\title{
Motivos de elección de carrera
}

\section{en mujeres estudiantes de profesiones de la salud}

\section{Health sciences careers choice reasons in women students.}

\author{
Elizabeth Verde Flota, Georgina Gallardo Hernández, Sandra Compeán Dardón, \\ Silvia Tamez González y Luis Ortiz-Hernández
}

Departamento de Atención a la Salud. Universidad Autónoma Metropolitana-Xochimilco, México

Objetivo: Analizar los motivos por los que mujeres estudiantes del área de la salud elijen sus carreras. Material y método: La recolección de información se realizó a través de encuestas de autorespuesta para estudiantes de nuevo ingreso en el año del 2004 de las carreras de medicina, enfermería, odontología y nutrición de la Universidad Autónoma Metropolitana Xochimilco, México. Solamente se consideraron a las alumnas cuya primera opción fueron las carreras antes señaladas. En total se aplicaron 437 encuestas. La variable independiente fue la internalización de estereotipos de género evaluada mediante el Inventario de Masculinidad y Feminidad. La variable dependiente fueron los motivos por los cuales las estudiantes habían elegido su carrera.

Resultados: Las mujeres de medicina tuvieron más rasgos masculinos que las de enfermería y odontología; mientras que las últimas tuvieron más características relacionadas con la sumisión. Las médicas con más frecuencia reportaron haber elegido su carrera por tener cualidades relacionadas con la masculinidad como la toma de decisiones o el prestigio social.
Correspondencia:

L. Ortiz Hernández

E. Verde Flota

Departamento de Atención a la Salud (UAM-X)

Calzada del Hueso 1100

Col. Villa Quietud

Coyoacán, México, 04960.

Tel.: 54-83-75-73

Fax: 54-83-72-18

E-mail: lortiz@correo.xoc.uam.mx everde@correo.xoc.uam.mx
Las nutriólogas con más frecuencia señalaron que eligieron su carrera pues les permitía aprender a mantenerse saludables. Las odontólogas indicaron que habían elegido su profesión debido a que les permite cuidar a sus hijos.

Conclusiones: La influencia de las normas de género en la trayectoria académica de las mujeres se ve reflejada en las características de las mujeres que estudian cada profesión y en sus motivos de elección de carrera.

Palabras clave: género, mujeres, estudiantes, elección de carrera, profesiones de la salud.

Objective: To analyze the reasons by which student women of the health area choice their careers. Material and method:. The harvesting of the information was made through surveys of autoresponse for entrance students in 2004 of the medicine, nursing, odontology and dietetics careers of the Universidad Autónoma Metropolitana - Xochimilco, Mexico. Only were considered the students whose first option was these careers. Altogether 437 surveys were applied. The independent variable was the internalization of gender stereotypes evaluated by means of the Inventory of Masculinidad and Feminidad. The dependent variable was the reasons by which the students had chosen their career.

Results: The women from medicine had more masculine characteristics than those of nursing and odontology; whereas the last ones had more characteristics related to the submission. The medical ones reported, with more frequency, to have chosen 
their career because it has qualities related to the masculinity as the decision making or social prestige. The dietitians with more frequency indicated that they chose their careers because it allowed them to learn to stay healthful. The odontology women indicated that they had chosen their profession because allowed themto take care of its children.

Conclusions: The influence of the gender norms in the academic trajectory of the women is reflected in the characteristics of the women that study each profession and in their motives of career selection.

Key words: gender; women; students; careers selection; health professions.

\section{INTRODUCCIÓN}

Actualmente existe interés por parte de las Instituciones de Educación Superior (IES) en reducir la deserción escolar. En el caso de las mujeres estudiantes, uno de los factores que pueden influir son los estereotipos y roles de género. La gran mayoría de los estudios sobre esta temática sólo se han limitado a documentar la distribución porcentual de hombres y mujeres ${ }^{1,2,3,4}$ y en otros en los que se ha reflexionado sobre el predominio de las mujeres en ciertas carreras ${ }^{2}$, estos hallazgos son explicados como consecuencia de la socialización de género pero sin apoyo empírico ${ }^{5}$. La única excepción es la enfermería sobre la cual se han realizado investigaciones sobre la influencia de los estereotipos de género ${ }^{6}$.

En la sociedad, el género toma forma en los estereotipos de género, los cuáles son creencias prescripciones, reglas, expectativas y atribuciones la mayoría de las veces implícitas de cómo deben comportarse hombres y mujeres, es decir, en los estereotipos de género se define lo que en cada sociedad se entiende como masculino o femenino ${ }^{7}$. Respecto a cómo deben comportarse los individuos, en los estereotipos de género está definido que los hombres deben ser masculinos, mientras que las mujeres se les exige ser femeninas; en otras palabras, se espera correspondencia exacta entre la biología de los individuos y la construcción sociocultural que representa el género ${ }^{8}$.

En el caso de las mujeres, el desarrollo del rol femenino se logra siendo un ser para otros, de los otros y realizado en los otros, es decir, la feminidad radica fundamentalmente en realizar un papel maternal ya sea como hijas, esposas o madres. "Las energías de cada mujer deben destinarse a satisfacer las necesidades vitales y los deseos de los otros, su trabajo, su pensamiento y su afectividad cumplen esa disposición" ${ }^{9}$. Es probable que el predominio de mujeres en ciertas profesiones sea producto del rol de género.

Por lo anterior este estudio pretende contribuir a la resolución de los siguientes planteamientos. ¿Las mujeres que eligen carreras femeninas (nutrición y enfermería), difieren de aquellas que estudian carreras tradicionalmente consideradas masculinas (medicina)? ¿La socialización del género que reciben las mujeres en sus familias influye en la elección de carrera?

\section{MATERIAL Y MÉTODOS}

La recolección de información se realizó a través de encuestas de autorespuesta para estudiantes de nuevo ingreso en el año 2004 en las licenciaturas de medicina, enfermería, odontología y nutrición de la Universidad Autónoma Metropolitana unidad Xochimilco. Solamente se consideraron a las alumnas cuya primera opción fueron las carreras antes señaladas. En total se aplicaron 437 encuestas.

La variable independiente fue la introyección de estereotipos de género estrechamente relacionados con identidad de género y la variable dependiente fue la percepción sobre la carrera. Para evaluar la identidad de género se aplicó el Inventario de masculinidad y feminidad (IMAFE), instrumento diseñado con el objetivo de medir roles de género de manera confiable y válida. Esta prueba tiene como origen el Inventario de Papeles Sexuales de Bem (BSRI), diseñado para la población estadounidense y validada en México entre 1983 y $1990^{10}$.

El IMAFE cuenta con cuatro escalas: a) masculinidad (confiado de si mismo, tomar decisiones, independiente, analítico, competitivo, racional, reflexivo, personalidad fuerte, hábil para dirigir, seguro de si mismo, autosuficiente, dispuesto a arriesgarse, valiente, atlético, maduro); b) feminidad (sensible a las necesidades de los demás, comprensivo, tierno, afectuoso, cariñoso, gusto por los niños, dulce, caritativo, de voz suave, compasivo, amigable); c) machismo (agresivo, dominante, autoritario, egoísta, materialista, frío, ambicioso, rudo, enérgico, incomprensivo, individualista, arrogante) y d) sumi- 
sión (indeciso, de personalidad débil, cobarde, sumiso, incapaz de planear, dependiente, influenciable, conformista, tímido, retraído, pasivo, simplista, resignado, inseguro de si mismo), cada escala con quince reactivos (en total 60) que expresan rasgos de personalidad. Las personas deben indicar en que medida tienen o no esos rasgos, para ello pueden elegir una opción en una escala de 1 (nunca o casi nunca soy así) a 7 (siempre o casi siempre soy así). Para construir cada escala se sumó la puntuación de los 15 reactivos que la conforman.

Para evaluar la socialización de los estereotipos de género se consideró relevante conocer la distribución de las tareas domésticas entre mujeres y hombres del núcleo familiar de cada estudiante. Se indagó la distribución de las siguientes tareas: limpieza de la casa, arreglo de camas y ropa, lavar y planchar, ir al mercado, preparación de los alimentos, servir comida, pago de servicios, arreglos de desperfectos de la vivienda y trabajar para sostener a la familia. Se esperaba que esta información pro- porcionara un acercamiento a los roles de género existentes en la familia de las(os) estudiantes.

También se indagó sobre los motivos por los que los estudiantes habían elegido la licenciatura que estaban estudiando. Los motivos considerados fueron: te interesa cuidar a las personas, es una profesión compatible con las expectativas personales, es una profesión que permite controlar la salud de las personas, es una profesión adecuada para tu sexo, en su familia había miembros que estudiaron la misma carrera, es una carrera en la que se aprende como mantenerse saludable, es una profesión en la que siempre hay trabajo, es una profesión que se ejerce libremente, es una profesión en dónde se toman decisiones importantes, es una profesión que permite el cuidado de los hijos, es una profesión con prestigio ó estatus social, es una profesión bien pagada, es una profesión en dónde se puede obtener puestos importantes y es una profesión compatible con las labores del hogar.

Para el análisis estadístico se evaluaron las dife-

Tabla 1. Puntuaciones de las subescalas del IMAFE y en rasgos específicos de mujeres estudiantes de diferentes carreras de la salud

\begin{tabular}{|c|c|c|c|c|c|c|}
\hline & Total & $\operatorname{Enf}(a)$ & Nutr (b) & Estom (c) & Med (d) & Sig \\
\hline Masculinidad & 72.1 & $69.7 d$ & 73.2 & 70.8 & $74.6 a$ & $*$ \\
\hline Analítica & 4.9 & $4.8 d$ & $5.1 \mathrm{c}$ & $4.5 b d$ & $5.3 a c$ & $* * *$ \\
\hline Hábil para dirigir & 4.4 & $4.0 d$ & 4.6 & 4.3 & $4.6 a$ & $*$ \\
\hline Racional & 5.0 & 4.6bd & $5.2 \mathrm{a}$ & $4.7 d$ & $5.5 \mathrm{ac}$ & $*$ \\
\hline Reflexiva & 5.3 & 5.3 & 5.5 & $5.1 d$ & $5.6 c$ & $* *$ \\
\hline Personalidad fuerte & 4.4 & $4.1 d$ & 4.4 & $4.1 d$ & $4.8 \mathrm{ac}$ & $* *$ \\
\hline Feminidad & 79.9 & 81.4 & 78.6 & 80.7 & 79.0 & \\
\hline Compasiva & 4.6 & 4.5 & 4.7 & 4.4 & 5.0 & + \\
\hline Sensible a necesidades de otros & 5.6 & 5.6 & 5.5 & $5.4 d$ & $5.8 c$ & + \\
\hline Caritativa & 5.2 & 5.3 & $4.8 \mathrm{c}$ & $5.3 b$ & 5.2 & * \\
\hline Cariñosa & 5.7 & 5.7 & 5.5 & 5.9 & 5.4 & + \\
\hline Tierna & 5.6 & $5.8 d$ & 5.3 & $5.8 d$ & $5.3 a c$ & $* *$ \\
\hline Dulce & 5.0 & 5.1 & 4.8 & 5.2 & 4.7 & + \\
\hline Le gustan los niños & 5.4 & $5.8 \mathrm{~d}$ & 5.3 & 5.6 & $5.1 a$ & $* *$ \\
\hline De voz suave & 4.4 & 4.4 & 4.2 & $4.8 d$ & $3.9 c$ & $* *$ \\
\hline Machismo & 44.4 & 43.1 & 45.7 & $42.3 d$ & $46.9 c$ & $* *$ \\
\hline Dominante & 3.8 & $3.4 d$ & 4.1 & 3.6 & $4.1 \mathrm{a}$ & $* *$ \\
\hline Individualista & 3.6 & 3.3 & 3.9 & 3.5 & 3.7 & + \\
\hline Materialista & 2.7 & $2.4 b$ & 3.0a & 2.7 & 2.7 & + \\
\hline Egoísta & 2.1 & 1.9 & 2.3 & 2.0 & 2.3 & $* *$ \\
\hline Fría & 2.6 & 2.6 & 2.7 & $2.2 d$ & $2.8 \mathrm{c}$ & $* *$ \\
\hline Sumisión & 37.7 & $41.4 \mathrm{bd}$ & $36.3 a$ & 37.5 & $36.4 a$ & $* *$ \\
\hline Sumisa & 2.5 & 3.obd & $2.3 a$ & 2.5 & $2.3 a$ & $* *$ \\
\hline Incapaz de planear & 2.1 & $2.5 \mathrm{bcd}$ & $1.9 a$ & $2.0 \mathrm{a}$ & $1.9 a$ & $* *$ \\
\hline Personalidad débil & 2.4 & 3.0bcd & $2.1 a$ & $2.3 a$ & $2.3 a$ & $* * *$ \\
\hline Pasiva & 3.8 & 4.3bd & $3.5 a$ & 4.0 & $3.5 a$ & $* * *$ \\
\hline Dependiente & 3.2 & 3.7bd & $3.0 \mathrm{a}$ & 3.3 & $2.9 a$ & $* *$ \\
\hline Retraída & 2.1 & $2.4 b$ & $1.9 a$ & 2.0 & 2.0 & * \\
\hline Tímida & 2.9 & $3.2 b$ & $2.6 a$ & 3.1 & 2.8 & $*$ \\
\hline
\end{tabular}

$+p<0.010, * p \leq 0.05, * * p \leq 0.01, * * * p \leq 0.001$. Abreviaturas: Enf, enfermería; Nutr, nutrición; Estom, estomatología; Med, medicina;

Sig, signifincancia. Los supraíndices muestran el grupo con que existió diferencias significativas (prueba de Tukey), a= enfermería, b= nutrición, $c=$ estomatología y $d=$ medicina. 
Tabla 2. Motivos de elección de carrera en mujeres estudiantes de carreras de la salud

\begin{tabular}{|c|c|c|c|c|c|}
\hline & $\begin{array}{r}\text { Enf } \\
\% \\
\end{array}$ & $\begin{array}{r}\text { Nutr } \\
\% \\
\end{array}$ & $\begin{array}{r}\text { Esto } \\
\% \\
\end{array}$ & $\begin{array}{r}\text { Med } \\
\% \\
\end{array}$ & Sig \\
\hline \multicolumn{6}{|c|}{ En familia hay otros con misma carrera } \\
\hline Nunca / en ocasiones & 67.8 & 94.7 & 84.5 & 76.4 & \multirow[t]{3}{*}{$* * *$} \\
\hline Casi siempre & 8.0 & 0.0 & 0.7 & 5.5 & \\
\hline Siempre & 24.1 & 5.3 & 14.8 & 18.2 & \\
\hline \multicolumn{6}{|c|}{ Se aprende como mantenerse saludable } \\
\hline Nunca / en ocasiones & 6.9 & 0.0 & 2.8 & 10.0 & \multirow[t]{3}{*}{$* * *$} \\
\hline Casi siempre & 29.9 & 6.4 & 21.0 & 21.8 & \\
\hline Siempre & 63.2 & 93.6 & 76.2 & 68.2 & \\
\hline \multicolumn{6}{|c|}{ Profesión que se ejerce libremente } \\
\hline Nunca / en ocasiones & 26.4 & 13.8 & 1.4 & 13.6 & \multirow[t]{3}{*}{$* * *$} \\
\hline Casi siempre & 35.6 & 28.7 & 31.3 & 32.7 & \\
\hline Siempre & 37.9 & 57.4 & 67.4 & 53.6 & \\
\hline \multicolumn{6}{|c|}{ Se toman decisiones importantes } \\
\hline Nunca / en ocasiones & 11.5 & 14.7 & 9.0 & 1.8 & \multirow[t]{3}{*}{$* * *$} \\
\hline Casi siempre & 26.4 & 29.5 & 27.1 & 7.2 & \\
\hline Siempre & 62.1 & 55.8 & 63.9 & 91.0 & \\
\hline \multicolumn{6}{|l|}{ Permite cuidar a los hijos } \\
\hline Nunca / en ocasiones & 29.9 & 12.6 & 9.2 & 50.0 & \multirow[t]{3}{*}{$* * *$} \\
\hline Casi siempre & 26.4 & 21.1 & 33.8 & 25.5 & \\
\hline Siempre & 43.7 & 66.3 & 57.0 & 25.5 & \\
\hline \multicolumn{6}{|c|}{ Profesión con prestigio ó estatus social } \\
\hline Nunca / en ocasiones & 29.9 & 27.4 & 14.7 & 25.2 & \multirow[t]{4}{*}{$* * *$} \\
\hline Casi siempre & 42.5 & 34.7 & 35.0 & 17.1 & \\
\hline Siempre & 27.6 & 37.9 & 50.3 & 57.7 & \\
\hline \multicolumn{5}{|l|}{ Profesión bien pagada } & \\
\hline Nunca / en ocasiones & 44.7 & 33.7 & 18.2 & 18.5 & \multirow[t]{4}{*}{ ** } \\
\hline Casi siempre & 36.5 & 46.3 & 49.0 & 47.7 & \\
\hline Siempre & 18.8 & 20.0 & 32.9 & 20.7 & \\
\hline \multicolumn{5}{|c|}{ Compatible con labores del hogar } & \\
\hline Nunca / en ocasiones & 61.6 & 26.3 & 52.8 & 87.4 & \multirow[t]{3}{*}{$* * *$} \\
\hline Casi siempre & 22.1 & 31.6 & 26.1 & 6.3 & \\
\hline Siempre & 16.3 & 42.1 & 21.1 & 6.3 & \\
\hline
\end{tabular}

rencias entre las mujeres de las cuatro carreras respecto a las puntuaciones en las cuatro escalas del IMAFE, la distribución intrafamiliar del trabajo doméstico y los motivos de elección de carrera, para lo cual se estimaron las pruebas de chi cuadrada (en el caso de variables ordinales o nominales) y el análisis de varianza (para las variables continuas). Se

Figura 1. Promedios de puntuaciones en la escala IMAFE de acuerdo a la carrera

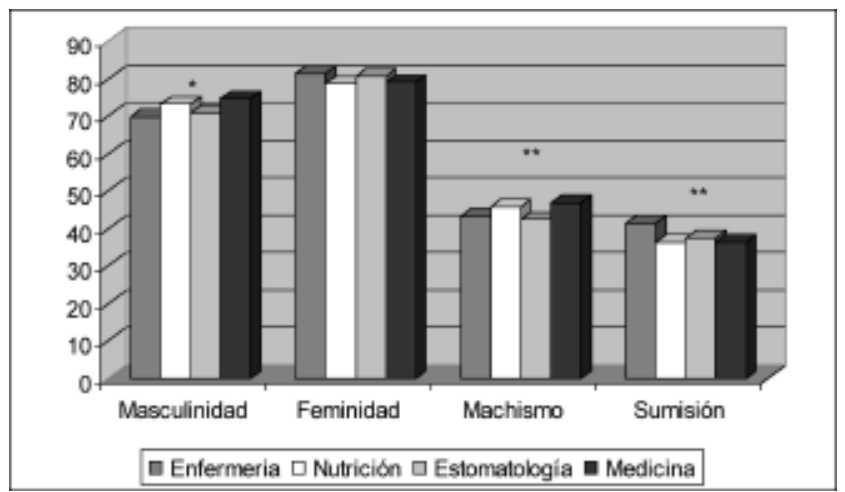

utilizó la prueba de Tukey para comparaciones múltiples con el fin de identificar las diferencias especificas que existieron entre licenciaturas en las puntuaciones del IMAFE. El procesamiento estadístico se realizó en el programa SPSS 10. En los cuadros sólo se muestran las comparaciones en las que existieron diferencias marginal $(\mathrm{p}<0.10)$ o estadísticamente significativas $(\mathrm{p}<0.05)$.

\section{RESULTADOS}

En la Tabla 1 y la figura 1 se presentan las puntuaciones de las subescalas del IMAFE y en los rasgos específicos de mujeres estudiantes de las diferentes carreras, con respecto a las estudiantes de enfermería, las estudiantes de medicina tuvieron puntuaciones más altas en la escala de masculinidad y de los siguientes rasgos: analítica, hábil para dirigir, racional y personalidad fuerte. En relación con las estudiantes de odontología las de medicina cuentan con mayores valores en los rasgos de: racional, reflexiva y personalidad fuerte. En la escala de 
Figura 2. Distribución (\%) de las mujeres estudiantes de acuerdo a algunos motivos de elección de carrera

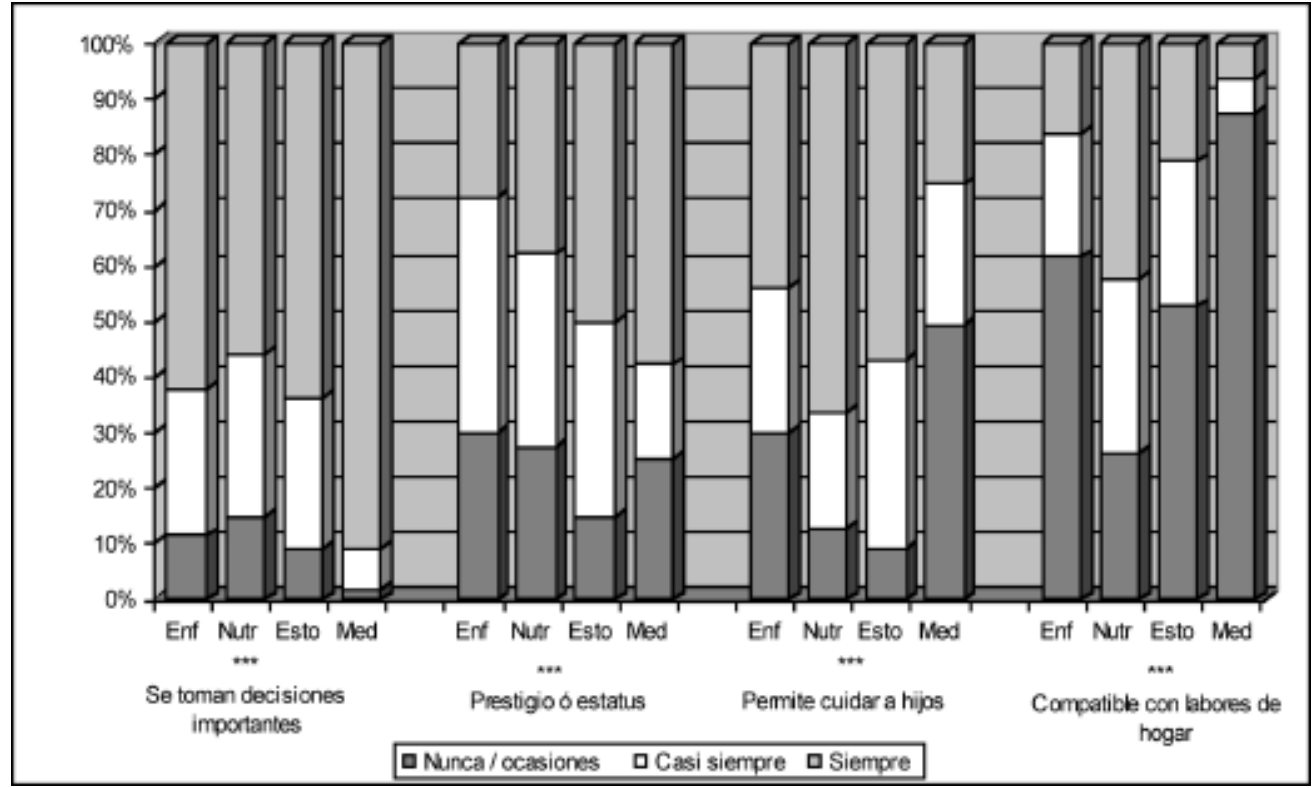

*** $p \leq 0.001$. Abreviaturas: Enf, enfermería; Nutr, nutrición; Estom, estomatología; Med, medicina

machismo las estudiantes de medicina tuvieron puntuaciones más altas en el rasgo dominante que los estudiantes de enfermería y más altas en la característica 'fría' que las de odontología. Con respecto a las estudiantes de nutrición presentaron rasgos de masculinidad estadísticamente significativa en relación con enfermería y odontología. Para la escala de sumisión las estudiantes de enfermería tuvieron puntuaciones más altas que las de nutrición y medicina; en el caso de odontología presentan puntuaciones relevantes en relación a medicina y nutrición. Las estudiantes de nutrición presentaron rasgos de masculinidad estadísticamente significativa en relación con enfermería y odontología. Respecto a la escala de feminidad no hubo diferencias entre las carreras.

No se encontraron diferencias entre las estudiantes de las cuatro licenciaturas respecto a la distribución intra-familiar del trabajo doméstico en función del sexo (los resultados no se muestran en cuadros).

La distribución de las estudiantes en función de los motivos de elección de carrera se muestra en el tabla 2 y la figura 2. Entre las estudiantes de enfermería fue más alta la proporción que reportó contar con un familiar con la misma profesión (24.1\%), seguida por medicina (18.2\%), odontología (14.8\%) y por último nutrición (5.3\%). Con respecto al ítem "se aprende a mantenerse saludable", las estudiantes de nutrición mostraron la frecuencia (93.6\%) en relación con las de enfermería (63.2\%). Considerar que la profesión permite cuidar a los hijos fue más frecuente entre las mujeres de nutrición y odontología (66.3\% y $57 \%$, respectivamente), mientras que enfermería y medicina tuvieron los porcentajes más bajos (43.7\% y 25.5\%). El prestigio ó estatus social de la profesión fue reportado con más frecuencia por las estudiantes de medicina $(57.7 \%)$ y odontología $(50.3 \%)$ respecto a las de enfermería $(27.6 \%)$ y nutrición (37.9\%). Más estudiantes de odontología $(32.9 \%)$ consideraron que su profesión es bien remunerada, mientras que la proporción más baja se observó en las de enfermería (18.8\%). Un porcentaje más alto de las alumnas de medicina (27\%) perciben que pueden acceder a puestos importantes, lo contrario sucede con estomatología (15.5\%). Más estudiantes de nutrición y odontología consideran que su futura profesión es compatible con las labores domésticas $(42.1 \%$ y $21.1 \%$ respectivamente), mientras que un porcentaje menor de alumnas de enfermería (16.3\%) y medicina (6.3\%), la visualizan de esta forma.

La relación de los motivos de elección de carrera con las escalas del IMAFE, se presenta en la tabla 3. Las mujeres que indicaron que habían elegido su licenciatura por el interés de cuidar otras personas, por que se aprende a mantenerse saludable, porque permite cuidar a los hijos y por que es compatible con las labores del hogar tuvieron puntuaciones más altas 
en la escala de feminidad respecto a las que reportaron que no habían elegido su profesión por esos motivos. Las estudiantes que eligieron su carrera por que siempre hay trabajo, es una profesión que se ejerce libremente y permite obtener puestos importantes tuvieron valores más altos en la escala de masculinidad con relación a las que no seleccionaron su licenciatura por dichos motivos. Las mujeres que indicaron que en la profesión que eligieron existe la posibilidad de tomar decisiones importantes y que tiene prestigio o status social tuvieron puntuaciones más altas en las escalas de feminidad y masculinidad.

\section{DISCUSIÓN}

En el contexto de las repercusiones de la internalización de los estereotipos de género sobre la esfera académica, las mujeres, en la actualidad, no existe

Tabla 3. Escalas IMAFE de acuerdo a los motivos de elección de carrera en mujeres estudiantes de carreras de la salud

\begin{tabular}{|c|c|c|c|c|c|c|c|c|}
\hline & \multicolumn{2}{|c|}{ Feminidad } & \multicolumn{2}{|c|}{ Sumisión } & \multicolumn{2}{|c|}{ Masculin. } & \multicolumn{2}{|c|}{ Machismo } \\
\hline & ÂX & Sig & ÂX & Sig & ÂX & Sig & ÂX & Sig \\
\hline \multicolumn{9}{|l|}{ Interesa cuidar a personas } \\
\hline Nunca / en ocasiones & 70.1 & $* * *$ & 39.0 & & 67.7 & $*$ & 43.7 & $* *$ \\
\hline Casi siempre & 75.2 & & 39.0 & & 70.9 & & 47.9 & \\
\hline Siempre & 82.3 & 37.2 & 72.9 & 43.3 & & & & \\
\hline \multicolumn{9}{|c|}{ Compatible c/ expectativas personales } \\
\hline Nunca / en ocasiones & 78.5 & $* *$ & 38.6 & $* * *$ & 67.3 & $* * *$ & 41.4 & \\
\hline Casi siempre & 75.7 & & 41.7 & & 67.3 & & 45.6 & \\
\hline Siempre & 80.8 & & 36.9 & & 73.3 & & 44.2 & \\
\hline \multicolumn{9}{|c|}{ Se controla la salud de as personas } \\
\hline Nunca / en ocasiones & 73.3 & $*$ & 36.6 & & 69.1 & & 45.5 & \\
\hline Casi siempre & 81.0 & & 36.5 & & 72.7 & & 43.6 & \\
\hline Siempre & 80.3 & & 38.1 & & 72.2 & & 44.5 & \\
\hline \multicolumn{9}{|c|}{ Se aprende a mantenerse saludable } \\
\hline Nunca / en ocasiones & 77.0 & ** & 37.8 & & 73.6 & & 48.5 & \\
\hline Casi siempre & 76.1 & & 37.3 & & 70.2 & & 43.8 & \\
\hline Siempre & 81.1 & & 37.8 & & 72.5 & & 44.3 & \\
\hline \multicolumn{9}{|l|}{ Siempre hay trabajo } \\
\hline Nunca / en ocasiones & 77.8 & & 36.2 & & 70.9 & ** & 43.2 & \\
\hline Casi siempre & 79.7 & & 38.3 & & 70.8 & & 44.4 & \\
\hline Siempre & 81.0 & & 37.7 & & 74.3 & & 44.8 & \\
\hline \multicolumn{9}{|c|}{ Profesión que se ejerce libremente } \\
\hline Nunca / en ocasiones & 76.4 & & 37.7 & & 68.7 & $*$ & 43.5 & \\
\hline Casi siempre & 80.1 & & 38.1 & & 71.3 & & 44.5 & \\
\hline Siempre & 80.7 & & 37.6 & & 73.3 & & 44.5 & \\
\hline \multicolumn{9}{|c|}{ Se toman decisiones importantes } \\
\hline Nunca / en ocasiones & 70.8 & $* * *$ & 41.3 & & 66.1 & $* * *$ & 45.9 & \\
\hline Casi siempre & 79.7 & & 37.5 & & 69.3 & & 42.7 & \\
\hline Siempre & 81.2 & & 37.3 & & 73.7 & & 44.7 & \\
\hline \multicolumn{9}{|l|}{ Permite cuidar a los hijos } \\
\hline Nunca / en ocasiones & 76.2 & $* *$ & 37.7 & & 70.2 & & 44.2 & \\
\hline Casi siempre & 80.0 & & 37.1 & & 71.8 & & 43.1 & \\
\hline Siempre & 81.8 & & 38.0 & & 73.3 & & 45.6 & \\
\hline \multicolumn{9}{|l|}{ Prestigio ó estatus social } \\
\hline Nunca / en ocasiones & 77.2 & $*$ & 36.4 & & 70.3 & $*$ & 42.6 & $* *$ \\
\hline Casi siempre & 79.9 & & 37.9 & & 70.9 & & 42.7 & \\
\hline Siempre & 81.4 & & 38.2 & & 73.8 & & 46.4 & \\
\hline \multicolumn{9}{|c|}{ Obtener puestos importantes } \\
\hline Nunca / en ocasiones & 78.9 & & 38.2 & & 70.8 & $* *$ & 43.6 & $* *$ \\
\hline Casi siempre & 80.7 & & 36.6 & & 71.4 & & 43.4 & \\
\hline Siempre & 80.6 & & 38.8 & & 75.7 & & 47.8 & \\
\hline \multicolumn{9}{|c|}{ Compatible con labores de hogar } \\
\hline Nunca / en ocasiones & 78.2 & + & 37.8 & & 71.0 & + & 44.6 & \\
\hline Casi siempre & 81.4 & & 37.3 & & 73.8 & & 43.2 & \\
\hline Siempre & 83.1 & & 38.0 & & 73.6 & & 45.0 & \\
\hline
\end{tabular}

$+\mathrm{p}<0.010, * \mathrm{p} \leq 0.05, * * \mathrm{p} \leq 0.01,{ }^{* * *} \mathrm{p} \leq 0.001$. Abreviaturas:ÂX, promedio; Sig, significancia 
ninguna restricción formal para que pueda elegir una ocupación considerada "masculina" y viceversa en el caso del varón; sin embargo, es claro que existe predominio de mujeres o varones en ciertas profesiones. "Si bien es cierto que los patrones de elección profesional de las mujeres han cambiado y que sus opciones se han diversificado, también lo es sólo para una minoría que deciden estudiar carreras largas, que implican un alto grado de dificultad, o que socialmente son consideradas como de mayor prestigio" ${ }^{11}$.

En este estudio el impacto del género en la elección de carrera de las estudiantes que ingresaron a la UAM-X en el 2004 se observó para el caso de medicina que sus rasgos predominantes son la masculinidad y el machismo, en el sentido de que las mujeres que eligen carreras consideradas masculinas tienden a presentar más rasgos masculinos con relación a aquellas que estudian carreras femeninas $^{3,12}$. Con las mujeres estudiantes de medicina sobresalen características masculinas y machistas y en consecuencia las labores domesticas y el cuidado de los hijos no las consideran como parte de su rol de género. De esta forma, las mujeres que eligen carreras "masculinas" (medicina) tienden a presentar más rasgos masculinos con relación a aquellas que estudian carreras denominadas "femeninas" ${ }^{3,12}$.

Las estudiantes de enfermería registraron los valores más altos en los rubros de feminidad, es decir, se perciben más compasivas, sensibles a las necesidades de los demás, caritativas, cariñosas, amigables, tiernas, dulces, les agradan los niños, entre otros. Con respecto a la sumisión, las estudiantes de enfermería ocuparon el mayor porcentaje en comparación con otras carreras. Aunado a lo anterior ellas también ocupan el primer lugar al haber elegido la carrera por tradición familiar en la enfermería, datos que nos sugieren que el cuidado de la salud, función socialmente atribuida a la mujer y posteriormente a las enfermeras al profesionalizarse esta actividad. Las características que marcaron de origen a la carrera de enfermería fue ser exclusiva para mujeres, ser auxiliar del médico, con formación rígida, disciplinaria, eminentemente técnica, centrada en la enfermedad, de bajo nivel educativo y diseñada por los mismos médicos, quienes hasta 1974 orientaron la carrera al saber "básico" de la medicina dejando a las mismas enfermeras que enseñaran propedéutica hospitalaria para un eficiente cuidado de los enfermos ${ }^{13}$. Lo anterior sugiere que el género interviene en la definición de ciertos puestos de trabajo, tanto femeninos como masculinos, propiciando la estereotipación sexual de las ocupaciones.
Las estudiantes de odontología se consideran femeninas, sensibles a las necesidades de los demás, caritativas, tiernas y de voz suave, características en nuestra sociedad del rol que desempeña la mujer, así mismo su profesión desde sus expectativas les permite hacer compatible el cuidado de los hijos con su profesión. Situación que es debido al tipo de ejercicio profesional que desarrolla, es decir, contar con un consultorio en la propia casa ${ }^{2}$ y la flexibilidad de horario permite tener tiempo de atender a su familia o balancear la vida familiar con su trabajo ${ }^{14}$.

En el caso de nutrición, las estudiantes cuentan con rasgos de masculinidad como hábil para dirigir, racional, así como rasgos femeninos: compasivas, caritativas, cariñosas, amigables, aunado a lo anterior los motivos de elección de carrera se relaciona con: compatible con las labores del hogar, adecuada para su sexo, mantenerse saludable, el cuidado de los hijos, características que tradicionalmente se les ha asignado a las mujeres. Pareciera que en el caso de esta disciplina se profesionalizaron actividades femeninas lo que la hace atractiva para su ejercicio profesional en mujeres que tienen que hacer compatible su rol de género y el ámbito público. Los hallazgos de este estudio en este aspecto podría llevar a futuros análisis desde la perspectiva de Bem con su concepto de androginia psicológico en donde los individuos manifiestan características tanto masculinas como femeninas.

Así mismo los resultados obtenidos con relación a la elección de carreras consideradas femeninas como enfermería y nutrición, así como en licenciaturas tipificadas en la etapa de transición hacia la feminización como medicina y odontología, muestran características concordantes con las reportadas en otros estudios, sin embargo también arrojan algunos datos novedosos. Así pues los rasgos de masculinidad y machismo son marcados en el caso de las estudiantes de medicina y nutrición, es decir, desde sus perspectivas se consideran con personalidad fuerte, con capacidad de reflexión, racional, analítica y hábil para dirigir; además de dominantes, individualistas, materialistas, egoístas y frías, según la escala de la IMAFE. Situación no esperada para el caso de nutrición en donde los rasgos de masculinidad y de machismo se encuentran presentes, lo que puede estar asociado con nuevas formas de construcción del rol de género. Es decir, una nueva cultura de los roles de género en los que asume su feminidad pero con diferentes concepciones de lo que implica ser mujeres. Otro aspecto interesante en cuanto a motivo de elección de carrera relacionados con profesión de prestigio, estatus social y toma de decisiones conside- 
rados convencionalmente como masculinos, en este estudio también se asociaron con rasgos femeninos.

Finalmente, se partió de la premisa de que la distribución de las tareas domésticas eran indicativas de la socialización de los roles de género; sin embargo, no se encontraron diferencias entre las mujeres de las diferentes licenciaturas. Es de llamar la atención la participación masculina en las tareas del hogar, pareciera que estas nuevas generaciones han empezado a introyectar estas tareas, sin embargo quedaría la pregunta sobre que actividades del trabajo doméstico están dispuestos a realizar.

\section{BIBLIOGRAFÍA}

1. Ramírez MA, Méndez O, Nigenda G, Vargas MM. Recursos humanos en los servicios de salud: Una perspectiva de género. Centro de Análisis Social y Económico. 2002.

2. González D. Las mujeres y la educación médica. Rev Mex Educ Med 1990; 1 (3199):162-167.

3. Hamby CL. The role of psychological androgyny in female student's dental career choices. J Dent Educ. 1982 Sep; 46(9):537-40.

4. Cardaci D. Educación nutricional: mujeres culpabilizando mujeres. En: mujeres y medicina, no. 4. México. UAMXochimilco, 1990.

5. Pérez-Gil SE. La perspectiva de género: una alternativa en los estudios de alimentación y nutrición. En: Arechiga J, Bertrán
M (coords.). Significación sociocultural de la variación morfológica. México; UNAM, 1997.

6. Verde E, et al. Equidad y salario: un estudio de mujeres enfermeras. Desarrollo Científico de Enfermería. México. 2004, (12):293-298.

7. Lara-Cantú MA. Masculinidad y femineidad. En: Consejo Nacional de Población. Antología de la Sexualidad Humana I, pp. 315 - 333. México: CONAPO - Grupo editorial Miguel Ángel Porrua, 1994.

8. Ortiz L, Granados JA. Violencia hacia bisexuales, lesbianas y homosexuales de la Cuidad de México. Revista Mexicana de Sociología 2003 (3): 265-303.

9. Lagarde M. Genero y feminismo. Desarrollo humano y democracia. Madrid: Editorial Horas y Horas, 1996.

10. Lara - Cantú MA. Inventario de Masculinidad - femineidad (IMAFE). México: Manual Moderno, 1993.

11. González D. Las mujeres y la educación medica. Rev Mex Educ Med 1990; 1 (3199): 162-167.

12. Orpett S. Roles, Careers and feminity in biomedicine. Women physicians and nurses in Japan. Soc Sci med 198622 (1):81-90.

13. Scarbecz Ma y Ross JA. Gender differences in first-year student's motivation to attend dental school. J Dent Educ. 2002 66 (8): 952-961.

14. Cárdenas JM, Zarate GR. La formación y la práctica social de la profesión de enfermería en México. En RIEENF, septiembre, 2001. Disponible en:

http://tone.udea.co/revista/sep2001/enf.\%20mexico,htm 